\title{
Riemann-Finsler Geometry with Applications to Information Geometry
}

\author{
Zhongmin Shen
}

August 11, 2005

\section{Introduction}

Information geometry has emerged from investigating the geometrical structure of a family of probability distributions, and has been applied successfully to various areas including statistical inference, control system theory and multiterminal information theory $[\mathrm{Am}][\mathrm{AmNa}]$. The purpose of this paper is to give a brief introduction to Information Geometry from a more general point of view using Riemann-Finsler geometry and spray geometry.

Consider a set $\mathcal{F}$ of objects such as $2 \mathrm{D} / 3 \mathrm{D}$ images, or probability distributions and etc. To measure the difference from one object to another in $\mathcal{F}$, one defines a function, $\mathcal{D}$, called a divergence, on the product space $\mathcal{F} \times \mathcal{F}$ with the following properties

$$
\mathcal{D}(p, q) \geq 0, \quad \text { equality holds if and only if } p=q .
$$

The number $\mathcal{D}(p, q)$ measures the "divergence" of $p$ from $q$. The pair $(\mathcal{F}, \mathcal{D})$ is called a divergence space. To allow a great generality, the divergence $\mathcal{D}$ is not required to satisfy the reversibility condition: $\mathcal{D}(p, q)=\mathcal{D}(q, p)$.

For a divergence space $(\mathcal{F}, \mathcal{D})$, the set $\mathcal{F}$ is uaually not finite-dimensional in any sense. In practice, one considers a family of objects in $\mathcal{F}$, parametrized in a domain of $\mathrm{R}^{n}$. Such a family is called a model of $(\mathcal{F}, \mathcal{D})$. More precisely, a model of a divergence space $(\mathcal{F}, \mathcal{D})$ is an $n$-dimensional $C^{\infty}$ manifold $M$ as an embedded subset of $\mathcal{F}$ with the induced divergence $D=\mathcal{D}_{\mid M}$. Thus, a model $(M, D)$ itself is also a divergence space.

Below are several examples.

Example 1.1 Let $(\mathcal{M}, d)$ be a metric space. Then $\mathcal{D}:=\frac{1}{2} d^{2}$ is a divergence. This divergence is reversible, i.e., $\mathcal{D}(p, q)=\mathcal{D}(q, p)$.

Example 1.2 Let $\Omega \subset \mathrm{R}^{n}$ be an open subset and $\psi=\psi(x)$ be a $C^{\infty}$ function on $\Omega$ with

$$
\frac{\partial^{2} \psi}{\partial x^{i} \partial x^{j}}(x)>0 .
$$


Then

$$
\psi(z)-\psi(x)-(z-x)^{i} \frac{\partial \psi}{\partial x^{i}}(x) \geq 0 .
$$

Define $D: \Omega \times \Omega \rightarrow[0, \infty)$ by

$$
D(x, z):=\psi(z)-\psi(x)-(z-x)^{i} \frac{\partial \psi}{\partial x^{i}}(x) .
$$

$D$ is a divergence on $\Omega$.

More sophiscated examples are from other fields in natural science, such as mathematical psychology [DzCo1]-[DzCo3].

Our goal is to apply differential geometry to study regular models and the induced information structures. The regularity of divergence spaces and information structures will be defined in the following sections.

\section{$2 \quad f$-divergences on probability distributions}

An important class of divergence spaces come from Probability Theory.

Let $\mathcal{X}=(\mathcal{X}, \mathcal{B}, \nu)$ be a measure space, where $\mathcal{X}$ is a set, $\mathcal{B}$ is a completely additive class consisting $\mathcal{X}$ and its subsets, and $\nu$ is a $\sigma$-finite measure on $(\mathcal{X}, \mathcal{B})$. Let $\mathcal{P}=\mathcal{P}(\mathcal{X})$ be the space of probability distributions on $\mathcal{X}$.

$$
\mathcal{P}(\mathcal{X}):=\left\{p: \mathcal{X} \rightarrow[0, \infty) \mid \int_{\mathcal{X}} p(r) d r=1\right\} .
$$

The space $\mathcal{P}$ is convex in the sense that

$$
\lambda p+(1-\lambda) q \in \mathcal{P}, \quad \text { if } p, q \in \mathcal{P} .
$$

There is a special family of divergences on $\mathcal{P}$. Let $f:(0, \infty) \rightarrow \mathrm{R}$ be a convex function with

$$
f(1)=0, \quad f^{\prime \prime}(1)=1 .
$$

Define $\mathcal{D}_{f}: \mathcal{P} \times \mathcal{P} \rightarrow \mathrm{R}$ by

$$
\mathcal{D}_{f}(p, q):=\int_{\mathcal{X}} p(r) f\left(\frac{q(r)}{p(r)}\right) d r, \quad p=p(r), q=q(r) \in \mathcal{P} .
$$

By Jensen's inequality, we have

$$
\mathcal{D}_{f}(p, q) \geq f\left(\int p(r) \frac{q(r)}{p(r)} d r\right)=f(1)=0,
$$

where the equality holds if and only if $p=q$. Thus $\mathcal{D}_{f}$ is indeed a divergence on $\mathcal{P}$. We call $\mathcal{D}_{f}$ the $f$-divergence following I. Csiszàr. The $f$-divergence plays an important role in statistics. 
There is a more special family of $f$-divergences on $\mathcal{P}$. For $\rho \in \mathrm{R}$, let

$$
f_{\rho}(t):=\left\{\begin{array}{cl}
\frac{4}{1-\rho^{2}}\left(\frac{1+t}{2}-t^{(1+\rho) / 2}\right) & \text { if } \rho \neq \pm 1 \\
t \ln t & \text { if } \rho=1 \\
\ln (1 / t) & \text { if } \rho=-1 .
\end{array}\right.
$$

We have

$$
f_{\rho}(1)=0, \quad f_{\rho}^{\prime}(1)=\frac{2}{\rho-1}, \quad f_{\rho}^{\prime \prime}(1)=1, \quad f_{\rho}^{\prime \prime \prime}(1)=\frac{\rho-3}{2} .
$$

For $\rho=0$,

$$
f_{0}(t)=4\left(\frac{1+t}{2}-\sqrt{t}\right) .
$$

The divergence $\mathcal{D}_{0}$ on $\mathcal{P}$ is given by

$$
\mathcal{D}_{0}(p, q)=4\left\{1-\int \sqrt{p(r) q(r)} d r\right\}=2 \int(\sqrt{p(r)}-\sqrt{q(r)})^{2} d r .
$$

We see that $d_{0}(p, q):=\sqrt{2 \mathcal{D}_{0}(p, q)}$ is a distance function. $d_{0}$ is called the Hellinger distance and $\mathcal{D}_{0}=\frac{1}{2} d_{0}^{2}$ the Hellinger divergence.

For $\rho=-1$,

$$
f_{-1}(t)=\ln (1 / t) .
$$

The divergence $\mathcal{D}_{-1}$ on $\mathcal{P}$ is given by

$$
\mathcal{D}_{-1}(p, q)=\int p(r) \ln \frac{p(r)}{q(r)} d r .
$$

$\mathcal{D}_{-1}$ is called the Kullback-Leibler divergence.

\section{$3 \quad$ Regular divergences}

Before we discuss regular divergences, let us first introduce Finsler metrics and H-functions.

Definition 3.1 A Finsler metric on a manifold $M$ is a scalar function $L=$ $L(x, y)$ on $T M$ with the following properties:

(L1) $L(x, y) \geq 0$, and equality holds if and only if $y=0$;

(L2) $L(x, \lambda y)=\lambda^{2} L(x, y), \lambda>0$;

(L3) $L(x, y)$ is $C^{\infty}$ on $T M \backslash\{0\}$, and for any $y \in T_{x} M \backslash\{0\}$,

$$
g_{i j}(x, y):=\frac{1}{2} L_{y^{i} y^{j}}(x, y)>0,
$$


For a Finsler metric $L$ on a manifold $M$, the function $F_{x}:=\left.\sqrt{L}\right|_{T_{x} M}$ can be viewed as a norm on $T_{x} M$. Indeed, it satisfies the triangle inequality

$$
F_{x}(u+v) \leq F_{x}(u)+F_{x}(v), \quad u, v \in T_{x} M
$$

But the reversibility $\left(F_{x}(-u)=F_{x}(u)\right)$ is not assumed.

Let $g=g_{i j}(x) d x^{i} \otimes d x^{j}$ be a Riemannian metric as a tensor in the trational notation. Then we get a scalar function $L$ on $T M$

$$
L=g_{i j}(x) y^{i} y^{j}, \quad y=\left.y^{i} \frac{\partial}{\partial x^{i}}\right|_{x}
$$

By the above definition, $L$ is a Finsler metric. Namely, Riemannian metrics are special Finsler metrics. Usually, we denote a Riemannian metric by the letter $g=g_{i j}(x) y^{i} y^{j}$. Riemannian metrics are the most important metrics and have been studied throughly in the last century.

Let $(M, L)$ be a Finsler manifold. For a curve $C$ parametrized by $c=c(t)$, $0 \leq t \leq 1$, the length of $C$ is defined by

$$
\mathcal{L}(C)=\int_{0}^{1} \sqrt{L\left(c(t), c^{\prime}(t)\right)} d t .
$$

Using the length structure, we can define a function $d=d(p, q)$ on $M \times M$ by

$$
d(p, q)=\inf L(C)
$$

where the infimum is taken over all curves from $p$ to $q$. The distance function $d$ satisfies

(a) $d(p, q) \geq 0$ with equality holds if and only if $p=q$;

(b) $d(p, q) \leq d(p, r)+d(r, q)$.

$d$ is called the distance function of $L$.

Definition 3.2 A $H$-function on a manifold $M$ is a scalar function $H=H(x, y)$ on $T M$ with the following properties:

(H1) $H(x, \lambda y)=\lambda^{3} H(x, y), \lambda>0$.

(H2) $H(x, y)$ is $C^{\infty}$ on $T M \backslash\{0\}$.

$\mathrm{H}$-functions are positively homogeneous functions of degree three. There are lots of $\mathrm{H}$-functions. If $L=L(x, y)$ is a Finsler metric on a manifold $M$, then the following function

$$
H:=L(x, y)^{3 / 2}
$$

is a $\mathrm{H}$-function on $M$. If $L=L(x, y)$ is a Finsler metric on an open subset $\Omega \subset \mathrm{R}^{n}$, then

$$
H:=\frac{1}{2} L_{x^{k}}(x, y) y^{k}
$$


is a $\mathrm{H}$-function on $\Omega$.

Let $d=d(p, q)$ be the distance function of a Finsler metric $L$ on $M$. Let

$$
D(p, q):=\frac{1}{2} d(p, q)^{2}, \quad p, q \in M .
$$

$D$ is a divergence on $M$. In general, the divergence $D$ is not $C^{\infty}$ along the diagonal $\Delta=\{(p, p) \in M \times M\}$ unless $L$ is Riemannian. Nevertheless we have the following

Lemma 3.3 If $D$ is the divergence of a Finsler metric $L$ on a manifold $M$, then at any point $p$, there is a local coordinate system $(U, \phi)$ in $M$ such that

$$
2 D\left(\phi^{-1}(x), \phi^{-1}(x+y)\right)=L(x, y)+\frac{1}{2} L_{x^{k}}(x, y) y^{k}+o\left(|y|^{3}\right) .
$$

Now we are ready to define regular divergences.

Definition 3.4 Let $M$ be a manifold. A divergence function $D$ on $M$ is said to be regular if in any local coordinate system $(U, \phi)$ at any point in $M$ (restricted to a smaller domain if necessary),

$$
2 D\left(\phi^{-1}(x), \phi^{-1}(x+y)\right)=L(x, y)+P(x, y)+o\left(|y|^{3}\right),
$$

where $L=L(x, y)$ is a Finsler metric on $U$ and $P=P(x, y)$ is a $C^{\infty}$ function on $T U \backslash\{0\}$ with

$$
P(x, \lambda y)=\lambda^{3} P(x, y), \quad \lambda>0 .
$$

The Finsler metrics $L$ in (8) form a global Finsler metric on $M$, while the functions $P$ in (8) do not form a global scalar function on $T M$. However, one can use $P$ to define a $H$-function on $M$.

Lemma 3.5 Let $D$ be a regular divergence on $M$. Let $L$ and $P$ be the local functions defined by (8) in a local coordinate system $(U, \phi)$. Then

$$
H:=P(x, y)-\frac{1}{2} L_{x^{k}}(x, y) y^{k}
$$

is a well-defined H-function on $M$.

Proof: Let $\bar{L}=\bar{L}(\bar{x}, \bar{y})$ and $\bar{P}=\bar{P}(\bar{x}, \bar{y})$ be the local functions defined by (8) in another local coordinate system $(\bar{U}, \bar{\phi})$. Let $\bar{x}=\bar{\phi} \circ \phi^{-1}$.

$$
\bar{x}(x+y)=\bar{x}+\bar{y}+\frac{1}{2} \frac{\partial^{2} \bar{x}}{\partial x^{i} \partial x^{j}}(x) y^{i} y^{j}+o\left(|y|^{2}\right),
$$

where

$$
\bar{y}=\frac{\partial \bar{x}}{\partial x^{i}} y^{i} .
$$


By comparing the expansions (8) in both coordinate systems, we get

$$
\begin{gathered}
L(x, y)=\bar{L}(\bar{x}, \bar{y}) . \\
P(x, y)=\bar{P}(\bar{x}, \bar{y})+\frac{1}{2} \bar{L}_{\bar{y}^{k}}(\bar{x}, \bar{y}) \frac{\partial^{2} \bar{x}}{\partial x^{i} \partial x^{j}}(x) y^{i} y^{j} .
\end{gathered}
$$

Differentiating (10) yields

$$
\frac{1}{2} L_{x^{k}}(x, y) y^{k}=\frac{1}{2} \bar{L}_{\bar{x}^{k}}(\bar{x}, \bar{y}) \bar{y}^{k}+\frac{1}{2} \bar{L}_{\bar{y}^{k}}(\bar{x}, \bar{y}) \frac{\partial^{2} \bar{x}}{\partial x^{i} \partial x^{j}}(x) y^{i} y^{j} .
$$

Substracting it from (11), we obtain

$$
P(x, y)-\frac{1}{2} L_{x^{k}}(x, y) y^{k}=\bar{P}(\bar{x}, \bar{y})-\frac{1}{2} \bar{L}_{\bar{x}^{k}}(\bar{x}, \bar{y}) \bar{y}^{k} .
$$

Therefore the above function $H$ is well-defined on $M$.

Q.E.D.

Now for a regular divergence $D$ we have the following local expansion

$$
2 D\left(\phi^{-1}(x), \phi^{-1}(x+y)\right)=L(x, y)+\frac{1}{2} L_{x^{k}}(x, y) y^{k}+H(x, y)+o\left(|y|^{3}\right) .
$$

By Lemma 3.3, we have the following

Proposition 3.6 If $D$ is the divergence of a Finsler metric $L$ on a manifold $M$, then it is regular with $H=0$.

Example 3.7 Let $\Omega$ be an open subset in a Minkowski space $\left(\mathrm{R}^{n},\|\cdot\|\right)$ and $\psi(y)=a_{i j k} y^{i} y^{j} y^{k}$. Let

$$
D\left(x, x^{\prime}\right):=\frac{1}{2}\left\|x^{\prime}-x\right\|^{2}+\frac{1}{2} \psi\left(x^{\prime}-x\right), \quad x, x^{\prime} \in \Omega .
$$

Using the natural coordinate system $\varphi(x)=x$, we have

$$
2 D(x, x+y)=\|y\|^{2}+\psi(y) .
$$

Thus $D$ is a regular divergence with

$$
L(x, y)=\|y\|^{2}, \quad H(x, y)=\psi(y) .
$$




\section{Sprays of Finsler metrics}

Every Finsler metric $L$ on a manifold $M$ induces a vector field on $T M$,

$$
\mathcal{G}:=y^{i} \frac{\partial}{\partial x^{i}}-2 \mathcal{G}^{i}(x, y) \frac{\partial}{\partial y^{i}}
$$

where

$$
\mathcal{G}^{i}(x, y):=\frac{1}{4} g^{i l}(x, y)\left\{L_{x^{k} y^{l}}(x, y) y^{k}-L_{x^{l}}(x, y)\right\}
$$

where $\left(g^{i j}(x, y)\right):=\left(g_{i j}(x, y)\right)^{-1}$. From $(13)$, one can see that

$$
\mathcal{G}^{i}(x, \lambda y)=\lambda^{2} \mathcal{G}^{i}(x, y), \quad \lambda>0 .
$$

$\mathcal{G}$ is a well-defined $C^{\infty}$ vector field on $T M \backslash\{0\}$. We call $\mathcal{G}$ the spray of $L$.

It is possible that two distinct Finsler metrics having the same spray. For example, if $L$ is an arbitrary Finsler metric on a manifold, then the metric $\tilde{L}:=k L$ has the same spray as $L$ for any positive constant $k$.

If $L=g_{i j}(x) y^{i} y^{j}$ is a Riemannian metric, then

$$
\mathcal{G}^{i}(x, y)=\frac{1}{2} \gamma_{j k}^{i}(x) y^{j} y^{k}, \quad \gamma_{j k}^{i}(x)=\gamma_{k j}^{i}(x)
$$

where

$$
\gamma_{j k}^{i}(x)=\frac{1}{2} g^{i l}(x)\left\{\frac{\partial g_{j l}}{\partial x^{k}}(x)+\frac{\partial g_{k l}}{\partial x^{j}}(x)-\frac{\partial g_{j k}}{\partial x^{l}}(x)\right\} .
$$

The local functions $\gamma_{j k}^{i}(x)$ are called the Christoffel symbols. Note that $\mathcal{G}^{i}$ are quadratic in $y$.

A Finsler metric $L$ is called a Berwald metric if its spray coefficients $\mathcal{G}^{i}=$ $\frac{1}{2} \gamma_{j k}^{i}(x) y^{j} y^{k}$ are quadratic in $y$. There are many non-Riemannian Berwald metrics. An important fact is that every Berwald metric has the same spray as a Riemannian metric. This is due to Z.I. Szabo.

If $c=c(t)$ is an integral curve of $\mathcal{G}$ in $T M \backslash\{0\}$, then the local coordinates $(x(t), y(t))$ of $c(t)$ satisfy

$$
\left.\dot{x}^{i} \frac{\partial}{\partial x^{i}}\right|_{c(t)}+\left.\dot{y}^{i}(t) \frac{\partial}{\partial y^{i}}\right|_{c(t)}=y^{i}(t) \frac{\partial}{\partial x^{i}}{ }_{c(t)}-\left.2 \mathcal{G}^{i}(x(t), y(t)) \frac{\partial}{\partial y^{i}}\right|_{c(t)} .
$$

We obtain that $y^{i}(t)=\dot{x}^{i}(t)$ and

$$
\ddot{x}^{i}(t)+2 \mathcal{G}^{i}(x(t), \dot{x}(t))=0 .
$$

Let $\sigma(t):=\pi(c(t))$ be the projection of $c=c(t)$ by $\pi: T M \rightarrow M$. The local coordinates of $\sigma(t)$ are $x(t)=\left(x^{i}(t)\right)$, which satisfy (16). Conversely, if a curve $\sigma=\sigma(t)$ satisafies (16), then the canonical lift $c(t)=\dot{\sigma}(t)$ in $T M$ is an integral curve of $\mathcal{G}$ such that $\sigma(t)=\pi(c(t))$.

Definition 4.1 A curve $\sigma$ in a Finsler manifold $(M, L)$ is called a geodesic if its canonical lift $c:=\dot{\sigma}$ in $T M \backslash\{0\}$ is an integral curve of the induced spray $\mathcal{G}$ by $L$. 


\section{Sprays}

The notion of sprays induced by a Finsler metric can be generalized.

Definition 5.1 Let $M$ be a manifold. A spray $G$ on $M$ is a vector field on the tangent bundle $T M$ such that in any standard local coordinate system $\left(x^{i}, y^{i}\right)$ in $T M$, it can be expressed in the following form:

$$
G=y^{i} \frac{\partial}{\partial x^{i}}-2 G^{i}(x, y) \frac{\partial}{\partial y^{i}}
$$

where $G^{i}(x, y)$ are $C^{\infty}$ functions of $\left(x^{i}, y^{i}\right)$ with $y \neq 0$ and

$$
G^{i}(x, \lambda y)=\lambda^{2} G^{i}(x, y), \quad \lambda>0 .
$$

The notion of geodesics can also be extended to sprays. A curve $\sigma(t)$ is called a geodesic of $G:=y^{i} \frac{\partial}{\partial x^{i}}-2 G^{i}(x, y) \frac{\partial}{\partial y^{2}}$ on a manifold $M$ if it satisfies the following system of equations:

$$
\ddot{x}^{i}(t)+2 G^{i}(x(t), \dot{x}(t))=0,
$$

where $x(t)=\left(x^{i}(t)\right)$ denotes the coordinates of $\sigma(t)$. Geodesics are also called paths. The collection of all paths of a spray is called a path structure.

A spray $G=y^{i} \frac{\partial}{\partial x^{i}}-2 G^{i}(x, y) \frac{\partial}{\partial y^{i}}$ is said to be affine, if in any local coordinate system,

$$
G^{i}(x, y)=\frac{1}{2} \Gamma_{j k}^{i}(x) y^{j} y^{k}, \quad \Gamma_{j k}^{i}(x)=\Gamma_{k j}^{i}(x) .
$$

By definition, a Finsler metric is a Berwald metric if and only if its spray is affine.

Every affine spray $G$ with coefficients $G^{i}(x, y)=\frac{1}{2} \Gamma_{j k}^{i}(x) y^{j} y^{k}, \Gamma_{j k}^{i}(x)=$ $\Gamma_{k j}^{i}(x)$, defines a connection $\nabla$ on $T M$,

$$
\nabla_{y} X:=\left.\left\{d X^{i}(y)+X^{j} \Gamma_{j k}^{i}(x) y^{k}\right\} \frac{\partial}{\partial x^{i}}\right|_{x},
$$

where $X=X^{i} \frac{\partial}{\partial x^{i}} \in C^{\infty}(T M)$ and $y=\left.y^{i} \frac{\partial}{\partial x^{i}}\right|_{x} \in T_{x} M . \quad \nabla$ is linear in the following sense:

$$
\begin{gathered}
\nabla_{\lambda y+\mu v} X=\lambda \nabla_{y} X+\mu \nabla_{v} X, \\
\nabla_{y}(X+Y)=\nabla_{y} X+\nabla_{y} Y, \\
\nabla_{y}(f X)=d f_{x}(y) X+f(x) \nabla_{y} X,
\end{gathered}
$$

where $y, v \in T_{x} M, f \in C^{\infty}(M)$ and $X, Y \in C^{\infty}(T M)$. It is torsion-free in the following sense:

$$
\nabla_{X} Y-\nabla_{Y} X=[X, Y]
$$


where $X, Y \in C^{\infty}(T M)$. Torsion-free linear connections are also called affine connections.

Every affine spray defines an affine connection by (18). Conversely, every affine connection $\nabla$ on $T M$ defines a spray by (17). Thus affine connections one-to-one correspond to affine sprays.

$$
\{\text { affine connections }\} \longleftrightarrow\{\text { affine sprays }\}
$$

Definition 5.2 A spray $G$ on a manifold is said to be flat if at every point, there is a standard local coordinate system $\left(x^{i}, y^{i}\right)$ in $T M$ such that $G=y^{i} \frac{\partial}{\partial x^{i}}$, i.e., $G^{i}=0$. In this case, $\left(x^{i}, y^{i}\right)$ is called an adapted coordinate system.

Flat sprays are very special affine sprays. If $G$ is flat, then in an adapted coordinate system, the geodesics of $G$ are linear, i.e., the coordinates $\left(x^{i}(t)\right)$ of every geodesic $\sigma(t)$ are in the following linear form:

$$
x^{i}(t)=a^{i} t+b^{i} .
$$

\section{Information structures}

By definotion, any regular divergence $D$ on a manifold $M$ induces a Finsler metric $L$ and a $H$-function. They can be obtained by the following formulas:

$$
L(x, y)=\lim _{\epsilon \rightarrow 0^{+}} \frac{2 D(c(0), c(\epsilon))}{\epsilon^{2}},
$$

where $c(t)$ is an arbitrary $C^{1}$ curve in $M$ with $c(0)=x$ and $c^{\prime}(0)=y$;

$$
H(x, y)=\lim _{\epsilon \rightarrow 0^{+}} \frac{2 D(\sigma(0), \sigma(\epsilon))-L(x, y) \epsilon^{2}}{\epsilon^{3}},
$$

where $\sigma=\sigma(t)$ is the geodesic with $\sigma(0)=x$ and $\dot{\sigma}(0)=y$.

Definition 6.1 An information structure on a manifold $M$ is a pair $\{L, H\}$, where $L=L(x, y)$ is a Finsler metric on $M$ and $H=H(x, y)$ is a H-function.

Every regular divergence induces an information structure. Conversely, every information structure is induced by a regular divergence as shown below.

Proposition 6.2 Let $(L, H)$ be an information structure on a manifold $M$. There is a regular divergence $D$ on $M$ such that the induced structure by $D$ is $\{L, H\}$.

Proof: Let $d$ denote the distance function of $L$ on $M$ For $p, q \in M$, define

$$
D(p, q)=\frac{1}{2} d(p, q)^{2}+\inf _{c(0)=p, c(1)=q} \int_{0}^{1} H\left(c(t), c^{\prime}(t)\right) d t,
$$

where the infimum is taken over all minimizing geodesic $c$ from $p$ to $q$. Then it is easy to verify that $D$ induces $\{L, H\}$.

Q.E.D. 


\section{The $\alpha$-sprays of an information structure}

Let $(L, H)$ be an information structure on a manifold $M$. Let $\mathcal{G}=y^{i} \frac{\partial}{\partial x^{i}}-2 \mathcal{G}^{i} \frac{\partial}{\partial y^{i}}$ be the spray of $L$. Using $H$, we can define a family of sprays $G_{\alpha}=y^{i} \frac{\partial}{\partial x^{i}}-$ $2 G_{\alpha}^{i}(x, y) \frac{\partial}{\partial y^{2}}$ by

$$
G_{\alpha}^{i}(x, y):=\mathcal{G}^{i}(x, y)+\frac{\alpha}{2} g^{i j}(x, y) H_{y^{j}}(x, y) .
$$

$G_{\alpha}$ is called the $\alpha$-spray of $(L, H)$. Our motivation to find a spray better than $\mathcal{G}$ so that the geodesics of the spray are simple. However, the rate of change of the divergence along any geodesic of the $\alpha$-spray is not sensitive to $\alpha$.

Lemma 7.1 Let $D$ be a regular divergence on a manifold $M$ and $(L, H)$ be the induced information structure and $G_{\alpha}$ be the $\alpha$-spray of $(L, H)$. Let $\sigma=\sigma(t)$ be a geodesic. Then for any geodesic $\sigma$ of $G_{\alpha}$,

$$
\frac{2 D\left(\sigma\left(t_{o}\right), \sigma\left(t_{o}+\epsilon\right)\right)}{d\left(\sigma\left(t_{o}\right), \sigma\left(t_{o}+\epsilon\right)\right)^{2}}=1+\frac{H(x, y)}{3 L(x, y)} \epsilon+o(\epsilon) .
$$

where $x=\sigma\left(t_{o}\right)$ and $y=\dot{\sigma}\left(t_{o}\right)$.

Proof: Let $\phi=\left(x^{i}\right)$ be a local coordindate system in $M$. Let $x(t):=\phi(\sigma(t))$ and $\Delta x:=x\left(t_{o}+\epsilon\right)-x\left(t_{o}\right)$. We have

$$
\begin{aligned}
\Delta x^{i} & =\dot{x}^{i}\left(t_{o}\right) \epsilon+\frac{1}{2} \ddot{x}^{i}\left(t_{o}\right) \epsilon^{2}+o\left(\epsilon^{2}\right) \\
& =y^{i} \epsilon-G_{\alpha}^{i}(x, y) \epsilon^{2}+o\left(\epsilon^{2}\right) .
\end{aligned}
$$

By the above identity, we have

$$
\begin{aligned}
L(x, \Delta x) & =L \epsilon^{2}-L_{y^{k}} G_{\alpha}^{k} \epsilon^{3}+o\left(\epsilon^{3}\right) \\
L_{x^{k}}(x, \Delta x) \Delta x^{k} & =L_{x^{k}} y^{k} \epsilon^{3}+o\left(\epsilon^{3}\right) \\
H(x, \Delta x) & =H(x, y) \epsilon^{3}+o\left(\epsilon^{3}\right) .
\end{aligned}
$$

It follows from (13) that

$$
L_{y^{k}} \mathcal{G}^{k}=\frac{1}{2} L_{x^{k}} y^{k}
$$

Then by (23) we obtain

$$
\begin{aligned}
2 D\left(\sigma\left(t_{o}\right), \sigma\left(t_{o}+\epsilon\right)\right)= & 2 D\left(\phi^{-1}(x), \phi^{-1}(x+\Delta x)\right) \\
= & L(x, \Delta x)+\frac{1}{2} L_{x^{k}}(x, \Delta x) \Delta x^{k} \\
& +H(x, \Delta x)+o\left(\Delta x^{3}\right) \\
= & L \epsilon^{2}-L_{y^{k}} G_{\alpha}^{k} \epsilon^{3}+\frac{1}{2} L_{x^{k}} y^{k} \epsilon^{3}
\end{aligned}
$$




$$
\begin{aligned}
& +H \epsilon^{3}+o\left(\epsilon^{3}\right) \\
= & L \epsilon^{2}-L_{y^{k}} G_{\alpha}^{k} \epsilon^{3}+L_{y^{k}} \mathcal{G}^{k} \epsilon^{3} \\
& +H \epsilon^{3}+o\left(\epsilon^{3}\right) \\
= & L \epsilon^{2}+(1-3 \alpha) H \epsilon^{3}+o\left(\epsilon^{3}\right) .
\end{aligned}
$$

By a similar argument, we have

$$
d\left(\sigma\left(t_{o}\right), \sigma\left(t_{o}+\epsilon\right)\right)^{2}=L \epsilon^{2}-3 \alpha H \epsilon^{3}+o\left(\epsilon^{3}\right) .
$$

Combining the above two expansions, we obtain (22).

Q.E.D.

Definition 7.2 An information structure $(L, H)$ on a manifold is said to be $\alpha$-flat for some $\alpha$ if the $\alpha$-spray $G_{\alpha}$ of $(L, H)$ is flat. $(L, H)$ is said to be flat if it is 1 -flat.

Let $(L, H)$ be an information structure on $M$. Let

$$
L^{*}(x, y):=L(x,-y), \quad H^{*}(x, y):=H(x,-y) .
$$

Then $\left(L^{*}, H^{*}\right)$ is an information structure on $M$ too. We call $\left(L^{*}, H^{*}\right)$ the dual information structure of $(L, H)$. The following lemma is trivial.

Lemma 7.3 Let $(L, H)$ be an information structure on a manifold $M$. Then

(i) $(L, H)$ is $\alpha$-flat if and only if $(L, \alpha H)$ is 1-flat.

(ii) $(L, H)$ is $\alpha$-flat if and only if the dual $\left(L^{*}, H^{*}\right)$ is $(-\alpha)$-flat.

Proof: We only prove (ii). Let $\left(L^{*}, H^{*}\right)$ be its dual structure of $(L, H)$. Let $G_{\alpha}$ and $G_{\alpha}^{*}$ denote the $\alpha$-sprays of $(L, H)$ and $\left(L^{*}, H^{*}\right)$, respectively. First we have

$$
\begin{gathered}
\mathcal{G}^{* i}(x, y)=\mathcal{G}^{i}(x,-y) . \\
H_{y^{j}}^{*}(x, y)=-H_{y^{j}}(x,-y) .
\end{gathered}
$$

Thus

$$
G_{-\alpha}^{i}(x, y)=G_{\alpha}^{i}(x,-y) .
$$

By this, it is easy to see that $(L, H)$ is $\alpha$-flat if and only if $\left(L^{*}, H^{*}\right)$ is $(-\alpha)$-flat.

Q.E.D.

Lemma 7.4 Let $(L, H)$ be an information structure on an manifold $M$. For some $\alpha \neq 0,(L, H)$ is $\alpha$-flat if and only if at any point there is a local coordinate system $\left(x^{i}\right)$ such that

$$
\begin{aligned}
& L_{x^{k} y^{l}} y^{k}=2 L_{x^{l}}, \\
& \alpha H=-\frac{1}{6} L_{x^{k}} y^{k} .
\end{aligned}
$$


Proof: Suppose that $(L, H)$ is $\alpha$-flat. By assumption, there is a standard coordinate system $\left(x^{i}, y^{i}\right)$ in which $G_{\alpha}^{i}(x, y)=0$ hold. It follows from (23) and (21) that

$$
H(x, y)=-\frac{1}{3 \alpha} L_{y^{k}}(x, y) \mathcal{G}^{k}(x, y)=-\frac{1}{6 \alpha} L_{x^{k}}(x, y) y^{k} .
$$

Thus

$$
\left.\mathcal{G}^{i}(x, y)=-\frac{\alpha}{2} g^{i l} x, y\right) H_{y^{l}}(x, y)=\frac{1}{12} g^{i l}(x, y)\left[L_{x^{k}}(x, y) y^{k}\right]_{y^{l}} .
$$

Comparing it with (13), we obtain (24).

Conversely, if $L$ satisfies (24), then the spray coefficients of $L$ are given by

$$
\mathcal{G}^{i}(x, y)=\frac{1}{4} g^{i l}(x, y) L_{x^{l}}(x, y) .
$$

By (24) and (25), we have

$$
\left.\frac{\alpha}{2} g^{i l} x, y\right) H_{y^{l}}(x, y)=-\frac{1}{12} g^{i l}(x, y)\left[L_{x^{k}}(x, y) y^{k}\right]_{y^{l}}=-\frac{1}{4} g^{i l}(x, y) L_{x^{l}}(x, y) .
$$

Thus

$$
\left.G_{\alpha}^{i}(x, y)=\mathcal{G}^{i}(x, y)+\frac{\alpha}{2} g^{i l} x, y\right) H_{y^{l}}(x, y)=0 .
$$

Thus the $\alpha$-spray $G_{\alpha}$ is flat.

Q.E.D.

\section{Dually flat Finsler metrics}

In virtue of Lemma 7.4, we make the following

Definition 8.1 A Finsler metric $L$ on a manifold $M$ is said to be locally dually flat if at any point, there is a local coordinate system $\left(x^{i}\right)$ in which $L=L(x, y)$ satisfies (24), i.e.,

$$
L_{x^{k} y^{l}} y^{k}=2 L_{x^{l}} .
$$

Such a local system is called an adapted local system. $L$ is said to be (globally) dually flat if there is a $\mathrm{H}$-function $H$ such that $(L, H)$ is 1 -flat, that is, at every point there is a local coordinate system $\left(x^{i}\right)$ in which $L=L(x, y)$ satisfies (26) and the following equation:

$$
L_{x^{k}} y^{k}=-6 H .
$$

If $L$ is a locally dually flat Finsler metric on a manifold $M$, then at any point, there is a local coordinate system $\left(x^{i}\right)$ in which the spray coefficients $\mathcal{G}^{i}$ of $L$ satisfy

$$
\mathcal{G}^{i}+\frac{1}{2} g^{i j} H_{y^{j}}=0,
$$

where $H:=-\frac{1}{6} L_{x^{k}} y^{k}$.

Let us first consider locally dually flat Riemannian metrics. 
Proposition 8.2 A Riemannian metric $g=g_{i j}(x) y^{i} y^{j}$ on a manifold $M$ is locally dually flat if and only if it can be locally expressed as

$$
g_{i j}(x)=\frac{\partial^{2} \psi}{\partial x^{i} \partial x^{j}}(x),
$$

where $\psi=\psi(x)$ is a local scalar function on $M$.

Proof: Assume that $g$ is locally dually flat. There is a local coordinate system $\left(x^{i}\right)$ in which $L:=g$ satisfies $(24)$.

$$
\frac{\partial g_{i l}}{\partial x^{k}}(x)+\frac{\partial g_{k l}}{\partial x^{i}}(x)=2 \frac{\partial g_{i k}}{\partial x^{l}}(x) .
$$

Permutating $i$ and $l$ yields

$$
\frac{\partial g_{i l}}{\partial x^{k}}(x)+\frac{\partial g_{i k}}{\partial x^{l}}(x)=2 \frac{\partial g_{k l}}{\partial x^{i}}(x) .
$$

Subtracting (30) from (31) yields

$$
\frac{\partial g_{i k}}{\partial x^{l}}(x)=\frac{\partial g_{k l}}{\partial x^{i}}(x) .
$$

Thus there is a function $\psi(x)$ such that (29) holds. The converse is trivial.

Q.E.D.

Example 8.3 Let $\Omega \subset \mathrm{R}^{n}$ be a strongly convex domain defined by a Minkowski norm $\phi(y)$ on $\mathrm{R}^{n}$

$$
\Omega:=\left\{y \in \mathrm{R}^{n} \mid \phi(y)<1\right\} .
$$

Define $\Theta(x, y)>0, y \neq 0$, by

$$
\Theta(x, y)=\phi(y+\Theta(x, y) x), \quad y \in T_{x} \Omega=\mathrm{R}^{n} .
$$

It is easy to verify that $\Theta(x, y)$ satisfies

$$
\Theta_{x^{k}}(x, y)=\Theta(x, y) \Theta_{y^{k}}(x, y) .
$$

Let

$$
L(x, y):=\Theta(x, y)^{2} .
$$

Using (33), one obtains

$$
\begin{gathered}
L_{x^{k}}=2 \Theta^{2} \Theta_{y^{k}}, \\
L_{x^{k} y^{l}} y^{k}=\left[2 \Theta^{2} \Theta_{y^{k}}\right]_{y^{l}} y^{k}=\frac{4}{3}\left[\Theta^{3}\right]_{y^{l}}=4 \Theta^{2} \Theta_{y^{l}}, \\
\frac{L_{x^{k}} y^{k}}{2 L} L_{y^{l}}=\frac{2 \Theta^{2}}{2 \Theta^{2}} \cdot 2 \Theta \Theta_{y^{l}}=2 \Theta \Theta_{y^{l}} .
\end{gathered}
$$

Thus $L$ satisfies (24). Namely, $L$ is dually flat. 
A Finsler metric $L$ on an open domain $\mathcal{U} \subset \mathrm{R}^{n}$ is called a Funk metric, if $F:=\sqrt{L}$ satisfies

$$
F_{x^{k}}=F F_{y^{k}} .
$$

Every Funk metric is projectively flat, i.e., the geodesics are straight lines, or equivalently,

$$
F_{x^{k} y^{\imath}} y^{k}=F_{x^{l}} .
$$

A Finsler metric $L$ is mutally dually flat and projectively flat if $F:=\sqrt{L}$ satisfies (34) and $L$ satisfies (26). It can be shown that every mutually dually flat and projectively flat Finsler metric must be a Funk metric up to a scaling [SY].

\section{Affine divergences and affine information struc- tures}

In general, a regular divergence $D: M \times M \rightarrow[0, \infty)$ is not $C^{\infty}$ along the diagonal $\Delta:=\{(x, x), x \in M\}$.

Definition 9.1 A regular divergence $D$ on a manifold $M$ is called an affine divergence if $D$ is a $C^{\infty}$ function on a neighborhood of the diagonal in $M \times M$.

Lemma 9.2 Let $D$ be a regular affine divergence on a manifold $M$. Then the induced information structure $(L, H)$ has the following properties:

(i) $L=g_{i j}(x) y^{i} y^{j}$ is Riemannian,

(ii) $H=H_{i j k}(x) y^{i} y^{j} y^{k}$.

Proof: Let

$$
D\left(x, x^{\prime}\right):=D\left(\phi^{-1}(x), \phi^{-1}\left(x^{\prime}\right)\right) .
$$

By assumption $D\left(x, x^{\prime}\right)$ is $C^{\infty}$ in $x, x^{\prime}$. Since $D(x, x)=0$, we have the following Taylor expansion:

$$
2 D(x, x+y)=g_{i j}(x) y^{i} y^{j}+\frac{1}{3} h_{i j k}(x) y^{i} y^{j} y^{k}+o\left(|y|^{3}\right),
$$

where

$$
g_{i j}(x):=\left.\frac{\partial^{2} D}{\partial x^{\prime i} \partial x^{\prime j}}\left(x, x^{\prime}\right)\right|_{x^{\prime}=x}, \quad h_{i j k}(x)=\left.\frac{\partial^{3} D}{\partial x^{\prime i} \partial x^{\prime j} \partial x^{\prime k}}\left(x, x^{\prime}\right)\right|_{x^{\prime}=x} .
$$

Let

$$
H_{i j k}(x):=\frac{1}{3} h_{i j k}(x)-\frac{1}{6}\left\{\frac{\partial g_{i j}}{\partial x^{k}}(x)+\frac{\partial g_{i k}}{\partial x^{j}}(x)+\frac{\partial g_{j k}}{\partial x^{i}}(x)\right\} .
$$

Then

$$
2 D(x, x+y)=g_{i j}(x) y^{i} y^{j}+\frac{1}{2} \frac{\partial g_{i j}}{\partial x^{k}}(x) y^{i} y^{j} y^{k}+H_{i j k}(x) y^{i} y^{j} y^{k}+o\left(|y|^{3}\right) .
$$

Thus $L=g_{i j}(x) y^{i} y^{j}$ and $H=H_{i j k}(x) y^{i} y^{j} y^{k}$ are the induced metric and $\mathrm{H}-$ function.

Q.E.D. 
Remark 9.3 For an affine divergence,

$$
\left.\frac{\partial^{2} D}{\partial x^{i} \partial x^{j}}\left(x, x^{\prime}\right)\right|_{x^{\prime}=x}=\left.\frac{\partial^{2} D}{\partial x^{\prime i} \partial x^{\prime j}}\left(x, x^{\prime}\right)\right|_{x^{\prime}=x} .
$$

Definition 9.4 An information structure $\{L, H\}$ on a manifold $M$ is said to be affine if (i) $L=g_{i j}(x) y^{i} y^{j}$ is Riemannian and (ii) $H=H_{i j k}(x) y^{i} y^{j} y^{k}$ is a homogeneous polynomial.

If $\{L, H\}$ is an affine information structure, then $\left(L^{*}, H^{*}\right)=(L,-H)$.

Lemma 9.5 For an affine divergence $D$ on a manifold $M$ and its dual $D^{*}$, the induced information structure $\{L, H\}$ by $D$ is dual to the induced information structure $\left\{L^{*}, H^{*}\right\}$ by $D^{*}$.

Proof: It suffices to prove that the induced information structure of $D^{*}$ is $\{L,-H\}$.

Q.E.D.

\section{$10 \alpha$-flat affine information structures}

We are particularly interested in $\alpha$-flat information structures. If an information structure is $\alpha$-flat, then the associated $\alpha$-spray is flat.

In this section we are going to study flat affine information structures, and show that an affine information structure $(L, H)$ is $\alpha$-flat if and only if its dual $\left(L^{*}, H^{*}\right)$ is $\alpha$-flat.

Lemma 10.1 Let $(L, H)$ be an affine information structure on a manifold $M$ and $\alpha \neq 0 .(L, H)$ is $\alpha$-flat if and only if there is a local coordinate system $\left(x^{i}\right)$ and a local function $\psi=\psi(x)$ such that

$$
\begin{aligned}
L(x, y) & =\frac{\partial^{2} \psi}{\partial x^{i} \partial x^{j}}(x) y^{i} y^{j}, \\
H(x, y) & =-\frac{1}{6 \alpha} \frac{\partial^{3} \psi}{\partial x^{i} \partial x^{j} \partial x^{k}}(x) y^{i} y^{j} y^{k} .
\end{aligned}
$$

Proof: Assume that $(L, H)$ is $\alpha$-flat. By Lemma 7.4, there is a local coordinate system $\left(x^{i}\right)$ such that

$$
L_{x^{k} y^{l}} y^{k}=2 L_{x^{l}}
$$

Plugging $g_{i j} y^{i} y^{j}$ for $L$ into the above equation, one can find a function $\psi(x)$ such that

$$
g_{i j}(x)=\frac{\partial^{2} \psi}{\partial x^{i} \partial x^{j}}(x) .
$$

It follows from (25) that

$$
H_{i j k}(x)=-\frac{1}{6 \alpha} \frac{\partial^{3} \psi}{\partial x^{i} \partial x^{j} \partial x^{k}}(x)
$$


Conversely, if $L=g_{i j}(x) y^{i} y^{j}$ and $H=H_{i j k}(x) y^{i} y^{j} y^{k}$ are given by (37) and (38) respectively, then $L$ satisfies (24) and $H$ satisfies (25). Thus $(L, H)$ is $\alpha$-flat.

Q.E.D.

Lemma 10.2 Let $(L, H)$ be an affine information structure on a manifold $M$ and $\alpha \neq 0$. Assume that in a local coordinate system $\left(x^{i}\right),(L, H)$ is given by (35) and (36) respectively. Let $x_{i}^{*}:=\frac{\partial \psi}{\partial x^{i}}(x)$ and

$$
\psi^{*}\left(x^{*}\right):=-\psi(x)+\sum_{i=1}^{n} x_{i}^{*} x^{i},
$$

Then in the new coordinate system $\left(x^{* i}\right)$, the dual information structure $\left(L^{*}, H^{*}\right)=$ $(L,-H)$ is given by

$$
\begin{aligned}
L^{*}\left(x^{*}, y^{*}\right) & =\frac{\partial^{2} \psi^{*}}{\partial x_{i}^{*} \partial x_{j}^{*}}\left(x^{*}\right) y_{i}^{*} y_{j}^{*}, \\
H^{*}\left(x^{*}, y^{*}\right) & =-\frac{1}{6 \alpha} \frac{\partial^{3} \psi^{*}}{\partial x_{i}^{*} \partial x_{j}^{*} \partial x_{k}^{*}}\left(x^{*}\right) y_{i}^{*} y_{j}^{*} y_{k}^{*} .
\end{aligned}
$$

Thus $\left(L^{*}, H^{*}\right)$ is $\alpha$-flat.

Proof: First by (35), we have

$$
\mathcal{G}^{i}=\frac{1}{4} g^{i k}(x) \frac{\partial^{3} \psi}{\partial x^{i} \partial x^{j} \partial x^{k}}(x) y^{i} y^{j} .
$$

By definition,

$$
g_{i j}^{*}(x)=g_{i j}(x), \quad H_{i j k}^{*}(x)=-H_{i j k}(x) .
$$

The $\alpha$-spray $G_{\alpha}^{*}$ of $\left(L^{*}, H^{*}\right)$ is given by

$$
\begin{aligned}
G_{\alpha}^{* i}(x, y) & =\mathcal{G}^{i}(x, y)-\frac{\alpha}{2} g^{i k} H_{y^{k}}(x, y) \\
& =\frac{1}{2} g^{i k}(x) \frac{\partial^{3} \psi}{\partial x^{i} \partial x^{j} \partial x^{k}}(x) y^{i} y^{j},
\end{aligned}
$$

where $\left(g^{i j}(x)\right):=\left(g_{i j}(x)\right)^{-1}$. That is, the Christofeffel symbols $\left(\Gamma_{\alpha}\right)_{j k}^{* i}$ of $G_{\alpha}^{*}$ are given by

$$
\left(\Gamma_{\alpha}\right)_{j k}^{* i}(x)=g^{i l}(x) \frac{\partial^{3} \psi}{\partial x^{j} \partial x^{k} \partial x^{l}}(x) .
$$

Our goal is to find another local coordinate system $\left(x_{i}^{*}\right)$ in which $G^{*}$ is trivial. Consider the following map

$$
x_{i}^{*}:=\frac{\partial \psi}{\partial x^{i}}(x) .
$$

Since the Jacobian of $x^{*}=x^{*}(x)$ is just $\left(g_{i j}(x)\right)$, this map is a local diffeomorphism which can serve as a coordinate transformation. Define $\psi^{*}$ in $\left(x_{i}^{*}\right)$ by (39). By a direct computation, we obtain

$$
\frac{\partial \psi^{*}}{\partial x_{k}^{*}}\left(x^{*}\right)=x^{k}
$$


Since $\left(L^{*}, H^{*}\right)$ is affine, we can express $L^{*}$ and $H^{*}$ in the new coordinate system $\left(X^{* i}\right)$ by $L^{*}=g^{* k l}\left(x^{*}\right) y_{k}^{*} y_{l}^{*}$ and $H^{*}=H^{* i j k}\left(x^{*}\right) y_{i}^{*} y_{j}^{*} y_{k}^{*}$. It is easy to show that

$$
g^{* k l}\left(x^{*}\right)=\frac{\partial^{2} \psi^{*}}{\partial x_{k}^{*} \partial x_{l}^{*}}\left(x^{*}\right),
$$

and

$$
\frac{\partial^{2} x_{i}^{*}}{\partial x^{j} \partial x^{k}}(x)-\frac{\partial x_{i}^{*}}{\partial x^{l}}(x)\left(\Gamma_{\alpha}\right)_{j k}^{* l}(x)=0 .
$$

Thus, in the local coordinate system $\left(x_{i}^{*}\right)$, the spray coefficients of $G_{\alpha}^{*}$ vanish. This implies that

$$
H^{* i j k}\left(x^{*}\right)=-\frac{1}{6 \alpha} \frac{\partial^{3} \psi^{*}}{\partial x_{i}^{*} \partial x_{j}^{*} \partial x_{k}^{*}}\left(x^{*}\right) .
$$

Q.E.D.

By the above lemmas, we get the following

Theorem 10.3 Let $\alpha \neq 0$. An affine information structure $(L, H)$ is $\alpha$-flat if and only if its dual $\left(L^{*}, H^{*}\right)$ is $\alpha$-flat.

\section{Dualistic affine connections}

We know that affine connections one-to-one correspond to affine sprays. An affine connection on a Riemannian manifold $(M, g)$ is said to be dualistic if the dual linear connection $\nabla^{*}$ with respect to $g$ is also affine. In this section we are going to characterize dualistic affine connections.

Let $L=g_{i j} y^{i} y^{j}$ be a Riemannian metric on a manifold $M$ and $g=g_{i j} d x^{i} \otimes$ $d x^{j}$ the associated inner product on tangent spaces. For a linear connection $\nabla$ on $M$, define $\nabla^{*}$

$$
g\left(\nabla_{Z}^{*} X, Y\right)+g\left(X, \nabla_{Z} Y\right)=Z[g(X, Y)],
$$

where $X, Y, Z \in C^{\infty}(T M)$. It is easy to see that $\nabla^{*}$ is a linear connection too. $\nabla^{*}$ is called the dual connection of $\nabla$ with respect to $g$. The concept of duality between two linear connections on a Riemannian manifold is introduced by S.-I. Amari and H. Nagaoka [AmNa].

An important phenomenon is that if a linear connection $\nabla$ is affine, the dual linear connection $\nabla^{*}$ (with respect to $g$ ) is not necessarily affine (i.e., it might not be torsion-free).

Theorem 11.1 Let $g$ be a Riemannian metric on a manifold $M$. Every polynomial H-function on $(M, g)$ determines a dualistic affine connection. Conversely, every dualistic affine connection $\nabla$ determines a polynomial $H$-function. The correspondence is canonical,

$$
\Gamma_{j k}^{i}(x)=\gamma_{j k}^{i}(x)+3 g^{i l} H_{j k l}(x),
$$


where $\Gamma_{j k}^{i}$ denote the Christoffel symbols of $\nabla$ and $\gamma_{j k}^{i}$ denote the Christoffel symbols of $g$.

Proof: Let $H$ be a polynomial H-function on a Riemannian manifold $(M, g)$. Let $\nabla$ and $\bar{\nabla}$ be the affine connections corresponding to the associated 1-sprays $G_{1}$ and $\bar{G}_{1}$ of $(g, H)$ and $(g,-H)$, respectively. Note that $(g,-H)$ is dual to $(g, H)$. We claim that $\nabla$ and $\bar{\nabla}$ satisfy

$$
g\left(\bar{\nabla}_{Z} X, Y\right)+g\left(X, \nabla_{Z} Y\right)=Z[g(X, Y)],
$$

Namely, $\bar{\nabla}$ is dual to $\nabla$ with respect to $g$.

Let $g=g_{i j}(x) y^{i} y^{j}$ and $H=H_{i j k}(x) y^{i} y^{j} y^{k}$. Let $\Gamma_{j k}^{i}(x)$ and $\bar{\Gamma}_{j k}^{i}(x)$ denote the Christoffel symbols of $G_{1}$ and $\bar{G}_{1}$ respectively. Let $\Gamma_{j k, i}(x):=g_{i l}(x) \Gamma_{j k}^{l}(x)$, $\bar{\Gamma}_{j k, i}(x):=g_{i l}(x) \bar{\Gamma}_{j k}^{l}(x)$, and etc. From (21), we have

$$
\begin{aligned}
& \Gamma_{j k, i}(x)=\gamma_{j k, i}(x)+3 H_{i j k}(x), \\
& \bar{\Gamma}_{i k, j}(x)=\gamma_{i k, j}(x)-3 H_{i j k}(x) .
\end{aligned}
$$

Adding (45) and (46) yields

$$
\bar{\Gamma}_{i k, j}(x)+\Gamma_{j k, i}(x)=\gamma_{i k, j}(x)+\gamma_{j k, i}(x)=\frac{\partial g_{i j}}{\partial x^{k}}(x) .
$$

(47) can be written as (44). That is $\bar{\nabla}=\nabla^{*}$ is the dual linear connection of $\nabla$ on $(M, g)$. By definition, $\nabla$ is dualistic.

Let $\nabla$ be a affine connection on $(M, g)$. Define $H_{i j k}$ by (45). Clearly,

$$
H_{i j k}=H_{i k j} .
$$

Let $\nabla^{*}$ be the dual linear connection. Let $\Gamma_{j k}^{* i}$ denote the Christoffel symbols of $\nabla^{*}$ and $\Gamma_{j k, l}^{*}=g_{i l} \Gamma_{j k}^{* i}$. Then

$$
\Gamma_{i k, j}^{*}(x)+\Gamma_{j k, i}(x)=\frac{\partial g_{i j}}{\partial x^{k}}(x)=\gamma_{i k, j}(x)+\gamma_{j k, i}(x) .
$$

It follows from (45) and (48) that

$$
\Gamma_{i k, j}^{*}(x)=\gamma_{i k, j}(x)-3 H_{i j k}(x) .
$$

Suppose $\nabla^{*}$ is affine, i.e, $\Gamma_{j k}^{* i}=\Gamma_{k j}^{* i}$. Then

$$
H_{i j k}=H_{k j i} .
$$

Thus $H_{i j k}$ is symmetric in $i, j, k$. We obtain a polynomial $\mathrm{H}$-function $H=$ $H_{i j k}(x) y^{i} y^{j} y^{k}$. By (45), we see that $H$ satisfies (43).

Q.E.D.

Since on a Riemannian manifold $(M, g)$, dualistic affine connections one-toone correspond to polynomial $H$-functions. We immediately obtain the following 
Theorem 11.2 (Amari) Let $\nabla$ and $\nabla^{*}$ be dual affine connections on a Riemannian manifold $(M, g)$. Then $\nabla$ is flat if and only if $\nabla^{*}$ is flat

Proof: Let $H$ be the polynomial $\mathrm{H}$-fuction coresponding to $\nabla$. Then $H^{*}:=-H$ is the polynomial $\mathrm{H}$-function corresponding to $\nabla^{*}$. Note that the spray of $(g, H)$ (resp. $\left.\left(g, H^{*}\right)\right)$ is the spray defined by $\nabla$ (resp. $\nabla^{*}$ ). Thus $\nabla$ is flat if and only if $(g, H)$ is 1-flat.; $(g, H)$ is 1-flat if and only if $\left(g, H^{*}\right)$ is 1-flat by Theoem 10.3; $\left(g, H^{*}\right)$ is 1 -flat if and only if $\nabla^{*}$ is flat.

Q.E.D.

\section{Statistical models}

Let $\mathcal{P}$ be a space of probability distributions on a measure space $\mathcal{X}$ and $\mathcal{D}$ a divergence on $\mathcal{P}$. A statistical model in $(\mathcal{P}, \mathcal{D})$ is a pair $(M, D)$, where $M$ is a finite $C^{\infty}$ manifold embedded in $\mathcal{P}$ and $D$ is the restriction of $\mathcal{D}$ on $M$. If $f$ is a function satisfying (2), then it defines the f-divergence $\mathcal{D}_{f}$ on $\mathcal{P}$ by (3).

In this section, we are going to prove that for any manifold $M \subset \mathcal{P}$, the induced divergence $D_{f}=\left.\mathcal{D}_{f}\right|_{M}$ is affine, namely, the induced metric $L=$ $g_{i j}(s) y^{i} y^{j}$ is Riemannian and the induced H-function $H=H_{i j k}(x) y^{i} y^{j} y^{k}$ is a polynomial.

Theorem 12.1 Let $f=f(t)$ be a function with $f(1)=0$ and $f^{\prime \prime}(1)=1$. For any regular statistical model $\left(M, D_{f}\right)$ of $\left(\mathcal{P}, \mathcal{D}_{f}\right)$, the induced information structure on $M$ is given by $\left(L_{f}, H_{f}\right)=(L, \rho N)$, where $\rho:=3+2 f^{\prime \prime \prime}(1)$, and

$$
\begin{aligned}
L & =\int_{\mathcal{X}}\left[y^{i} \frac{\partial}{\partial x^{i}} \ln p\right]^{2} p d r \\
N & =\frac{1}{6} \int_{\mathcal{X}}\left[y^{i} \frac{\partial}{\partial x^{i}} \ln p\right]^{3} p d r .
\end{aligned}
$$

The $\alpha$-spray $G_{\alpha, \rho}$ of $D_{f}$ is given by $G_{\alpha, \rho}^{i}=\bar{G}^{i}+(\rho \alpha+1) A^{i}$, where

$$
\begin{aligned}
\bar{G}^{i} & =\frac{g^{i l}(x)}{2} \int_{\mathcal{X}}\left[y^{i} y^{j} \frac{\partial^{2}}{\partial x^{i} \partial x^{j}} \ln p\right] \frac{\partial}{\partial x^{l}} p d r \\
A^{i} & =\frac{g^{i l}(x)}{4} \int_{\mathcal{X}}\left[y^{i} \frac{\partial}{\partial x^{i}} \ln p\right]^{2} \frac{\partial}{\partial x^{l}} p d r .
\end{aligned}
$$

Proof: The natural embedding $M \rightarrow \mathcal{P}$ is given by $x \rightarrow p=p(r ; x)$. Let $D(x, z):=D_{f}(p(r ; x), p(r ; z))$, i.e.,

$$
D(x, z):=\int_{\mathcal{X}} p(r ; x) f\left(\frac{p(r ; z)}{p(r ; x)}\right) d r .
$$

We have

$$
2 D(x, x+y)=\left.\frac{\partial^{2} D}{\partial z^{i} \partial z^{j}}\right|_{z=x} y^{i} y^{j}+\left.\frac{1}{3} \frac{\partial^{3} D}{\partial z^{i} \partial z^{j} \partial z^{k}}\right|_{z=x} y^{i} y^{j} y^{k}+o\left(|y|^{3}\right) .
$$


By a direct computation, we obtain

$$
\begin{aligned}
\left.D\right|_{z=x} & =0 \\
\left.\frac{\partial D}{\partial z^{i}}\right|_{z=x} y^{i} & =0 \\
\left.\frac{\partial^{2} D}{\partial z^{i} \partial z^{j}}\right|_{z=x} y^{i} y^{j}= & \int_{\mathcal{X}}\left[y^{i} \frac{\partial}{\partial x^{i}} \ln p\right]^{2} p d r \\
\left.\frac{\partial^{3} D}{\partial z^{i} \partial z^{j} \partial z^{k}}\right|_{z=x} y^{i} y^{j} y^{k}= & \frac{\rho}{2} \int_{\mathcal{X}}\left[y^{i} \frac{\partial}{\partial x^{i}} \ln p\right]^{3} p d r \\
& +\frac{3}{2}\left\{-\int_{\mathcal{X}}\left[y^{i} \frac{\partial}{\partial x^{i}} \ln p\right]^{3} p+2\left[y^{i} y^{j} \frac{\partial^{2}}{\partial x^{i} \partial x^{j}} p\right]\left[y^{k} \frac{\partial}{\partial x^{k}} \ln p\right]\right\} d r
\end{aligned}
$$

Let

$$
L:=\int_{\mathcal{X}}\left[y^{i} \frac{\partial}{\partial x^{i}} \ln p\right]^{2} p d r
$$

Then

$$
\begin{aligned}
L_{x^{k}} y^{k}= & \int_{\mathcal{X}}\left[y^{i} \frac{\partial}{\partial x^{i}} \ln p\right]^{3} p d r \\
& +2 \int_{\mathcal{X}}\left[y^{k} \frac{\partial}{\partial x^{k}} \ln p\right]\left[y^{i} y^{j} \frac{\partial^{2}}{\partial x^{i} \partial x^{j}} \ln p\right] p d r \\
= & -\int_{\mathcal{X}}\left\{\left[y^{i} \frac{\partial}{\partial x^{i}} \ln p\right]^{3} p\right. \\
& \left.+2\left[y^{i} y^{j} \frac{\partial^{2}}{\partial x^{i} \partial x^{j}} p\right]\left[y^{k} \frac{\partial}{\partial x^{k}} \ln p\right]\right\} d r .
\end{aligned}
$$

Let

$$
N:=\frac{1}{6} \int_{\mathcal{X}}\left[y^{i} \frac{\partial}{\partial x^{i}} \ln p\right]^{3} p d r
$$

We obtain

$$
2 D(x, x+y)=L(x, y)+\frac{1}{2} L_{x^{k}}(x, y) y^{k}+\rho N(x, y)+o\left(|y|^{3}\right) .
$$

Thus $D_{f}$ is regular and the induced information structure $\left(L_{f}, H_{f}\right)=(L, \rho N)$ is affine.

Let $\mathcal{G}=y^{i} \frac{\partial}{\partial x^{i}}-2 \mathcal{G}^{i} \frac{\partial}{\partial y^{i}}$ denote the induced spray of $L$ and $G_{\alpha, f}=y^{i} \frac{\partial}{\partial x^{i}}-$ $2 G_{\alpha, \rho}^{i} \frac{\partial}{\partial y^{i}}$ be the $\alpha$-spray of $D_{f}$. Without much difficulty, we obtain

$$
\begin{aligned}
G_{\alpha, \rho}^{i}= & \mathcal{G}^{i}(x, y)+\frac{\rho \alpha}{2} g^{i l}(x) N_{y^{l}}(x, y) \\
= & (\rho \alpha+1) \frac{g^{i l}(x)}{4} \int_{\mathcal{X}}\left[y^{i} \frac{\partial}{\partial x^{i}} \ln p\right]^{2} \frac{\partial}{\partial x^{l}} p(r ; x) d r \\
& +\frac{g^{i l}(x)}{2} \int_{\mathcal{X}}\left[y^{i} y^{j} \frac{\partial^{2}}{\partial x^{i} \partial x^{j}} \ln p(r ; x)\right] \frac{\partial}{\partial x^{l}} p d r
\end{aligned}
$$


This gives a formula for $G_{\alpha, \rho}$.

Q.E.D.

Now let us express $L$ and $N$ in a different form. Observe that

$$
\begin{aligned}
L & =\int_{\mathcal{X}} y^{j} \frac{\partial}{\partial x^{j}}\left\{\left[y^{i} \frac{\partial}{\partial x^{i}} \ln p\right] p\right\} d r-\int_{\mathcal{X}}\left[y^{i} y^{j} \frac{\partial^{2}}{\partial x^{i} \partial x^{j}} \ln p\right] p d r \\
& =\int_{\mathcal{X}} y^{i} y^{j} \frac{\partial^{2}}{\partial x^{i} \partial x^{j}} p d r-\int_{\mathcal{X}}\left[y^{i} y^{j} \frac{\partial^{2}}{\partial x^{i} \partial x^{j}} \ln p\right] p d r \\
& =y^{i} y^{j} \frac{\partial^{2}}{\partial x^{i} \partial x^{j}} \int_{\mathcal{X}} p d r-\int_{\mathcal{X}}\left[y^{i} y^{j} \frac{\partial^{2}}{\partial x^{i} \partial x^{j}} \ln p\right] p d r \\
& =-\int_{\mathcal{X}}\left[y^{i} y^{j} \frac{\partial^{2}}{\partial x^{i} \partial x^{j}} \ln p\right] p d r .
\end{aligned}
$$

This gives

$$
L=-\int_{\mathcal{X}}\left[y^{i} y^{j} \frac{\partial^{2}}{\partial x^{i} \partial x^{j}} \ln p\right] p d r
$$

By a similar argument, we obtain

$$
\begin{aligned}
6 N= & y^{k} \frac{\partial}{\partial x^{k}} \int_{\mathcal{X}}\left[y^{i} \frac{\partial}{\partial x^{i}} \ln p\right]^{2} p d r \\
& -2 \int_{\mathcal{X}}\left[y^{i} y^{j} \frac{\partial^{2}}{\partial x^{i} \partial x^{j}} \ln p\right]\left[y^{k} \frac{\partial}{\partial x^{k}} p\right] d r \\
= & y^{k} \frac{\partial}{\partial x^{k}} \int_{\mathcal{X}}\left[y^{i} \frac{\partial}{\partial x^{i}} \ln p\right]\left[y^{j} \frac{\partial}{\partial x^{j}} p\right] d r \\
& -2 y^{k} \frac{\partial}{\partial x^{k}} \int_{\mathcal{X}}\left[y^{i} y^{j} \frac{\partial^{2}}{\partial x^{i} \partial x^{j}} \ln p\right] p d r \\
& +2 \int_{\mathcal{X}}\left[y^{i} y^{j} y^{k} \frac{\partial^{3}}{\partial x^{i} \partial x^{j} \partial x^{k}} \ln p\right] p d r \\
= & -3 y^{k} \frac{\partial}{\partial x^{k}} \int_{\mathcal{X}}\left[y^{i} y^{j} \frac{\partial^{2}}{\partial x^{i} \partial x^{j}} \ln p\right] p d r \\
& +2 \int_{\mathcal{X}}\left[y^{i} y^{j} y^{k} \frac{\partial^{3}}{\partial x^{i} \partial x^{j} \partial x^{k}} \ln p\right] p d r .
\end{aligned}
$$

This gives

$$
\begin{aligned}
N= & \frac{1}{3} \int_{\mathcal{X}}\left[y^{i} y^{j} y^{k} \frac{\partial^{3}}{\partial x^{i} \partial x^{j} \partial x^{k}} \ln p\right] p d r \\
& -\frac{1}{2} y^{k} \frac{\partial}{\partial x^{k}} \int_{\mathcal{X}}\left[y^{i} y^{j} \frac{\partial^{2}}{\partial x^{i} \partial x^{j}} \ln p\right] p d r .
\end{aligned}
$$

\section{Exponential family of distributions}

In this section, we will consider the exponential family of probability distributions, on which the $\alpha$-spray of $D_{f}$ with $\rho \alpha=-1$ is flat. 
Definition 13.1 A manifold $M$ in $\mathcal{P}$ is called an exponential manifold if it is covered by injections

$$
\varpi: \Omega \subset \mathrm{R}^{n} \rightarrow M,
$$

such that $p:=\varpi(x) \in \mathcal{P}$ is in the following form

$$
p(r ; x)=\exp \left[x^{i} f_{i}(r)+k(r)-\psi(x)\right], \quad r \in \mathcal{X} .
$$

Observe that the integral

$$
\int_{\mathcal{X}} \frac{\partial p}{\partial x^{i}} d r=0
$$

This implies that

$$
\frac{\partial \psi}{\partial x^{i}}(x)=\int_{\mathcal{X}} p(r ; x) f_{i}(r) d r .
$$

The Kullback-Leibler divergence $D_{K L}$ on $M$ is the $f$-divergence with $f(t)=$ $\ln (1 / t)$. We have

$$
\begin{aligned}
D_{K L}\left(p(r ; x), p\left(r ; x^{\prime}\right)\right) & =\int p(r ; x)\left[\psi\left(x^{\prime}\right)-\psi(x)-\left(x^{\prime}-x\right)^{i} f_{i}(r)\right] d r \\
& =\psi\left(x^{\prime}\right)-\psi(x)-\left(x^{\prime}-x\right)^{i} \frac{\partial \psi}{\partial x^{i}}(x) .
\end{aligned}
$$

The pull-back of $D_{K L}$ onto $\Omega$ is given by

$$
D_{K L}\left(x, x^{\prime}\right)=\psi\left(x^{\prime}\right)-\psi(x)-\left(x^{\prime}-x\right)^{i} \frac{\partial \psi}{\partial x^{i}}(x) .
$$

Proposition 13.2 Let $M$ be the exponential family of distributions in the form (56). The induced information structure of $D_{f}$ is given by $\left(L_{f}, H_{f}\right)=(L, \rho N)$, $\rho=3+2 f^{\prime \prime \prime}(1)$, and

$$
\begin{aligned}
L & =\frac{\partial^{2} \psi}{\partial x^{i} \partial x^{j}}(x) y^{i} y^{j} \\
N & =\frac{1}{6} \frac{\partial^{3} \psi}{\partial x^{i} \partial x^{j} \partial x^{k}}(x) y^{i} y^{j} y^{k}
\end{aligned}
$$

Proof: Note that

$$
\ln p(r ; x)=x^{i} f_{i}(r)+k(r)-\psi(x) .
$$

It follows from (54) that

$$
L(x, y)=\int_{\mathcal{X}}\left[y^{i} y^{j} \frac{\partial^{2} \psi}{\partial x^{i} \partial x^{j}}(x)\right] p(r ; x) d r=y^{i} y^{j} \frac{\partial^{2} \psi}{\partial x^{i} \partial x^{j}}(x) .
$$

Then the spray coefficients of $L$ are given by

$$
\mathcal{G}^{i}=\frac{1}{4} g^{i k} \frac{\partial^{2} \psi}{\partial x^{i} \partial x^{j} \partial x^{k}}(x) y^{i} y^{j} .
$$


It follows from (55) that

$$
\begin{aligned}
N(x, y)= & -\frac{1}{3} \int_{\mathcal{X}}\left[y^{i} y^{j} y^{k} \frac{\partial^{3} \psi}{\partial x^{i} \partial x^{j} \partial x^{k}}(x)\right] p(r ; x) d r \\
& +\frac{1}{2} y^{k} \frac{\partial}{\partial x^{k}} \int_{\mathcal{X}}\left[y^{i} y^{j} \frac{\partial^{2} \psi}{\partial x^{i} \partial x^{j}}(x)\right] p(r ; x) d r \\
= & \frac{1}{6} y^{i} y^{j} y^{k} \frac{\partial^{3} \psi}{\partial x^{i} \partial x^{j} \partial x^{k}}(x) .
\end{aligned}
$$

Q.E.D.

By Lemma 10.1, we obtain the following

Corollary 13.3 Let $M$ be the exponential family of distributions in the form (56). Let $\left(L_{f}, H_{f}\right)$ be the information structure induced by the $f$-divergence. When $\rho \alpha=-1,\left(L_{f}, H_{f}\right)$ is $\alpha$-flat, namely, the $\alpha$-spray of $\left(L_{f}, H_{f}\right)$ is flat.

Proof: The $\alpha$-spray is given by

$$
G_{\alpha, \rho}^{i}=\mathcal{G}^{i}+\frac{\rho \alpha}{2} g^{i k} N_{y^{k}}=\frac{\rho \alpha+1}{4} g^{i k} \frac{\partial^{3} \psi}{\partial x^{i} \partial x^{j} \partial x^{k}}(x) y^{i} y^{j} .
$$

If $\rho \alpha=-1$, then the induced information structure $\left(L_{f}, H_{f}\right)$ is $\alpha$-flat. Q.E.D.

Example 13.4 Consider the family $M$ of Gaussian probability distributions with mean $\mu$ and variance $\sigma^{2}$ :

$$
p\left(r ; \mu, \sigma^{2}\right)=\frac{1}{\sqrt{2 \pi} \sigma} \exp \left[-\frac{(r-\mu)^{2}}{2 \sigma^{2}}\right] .
$$

We can reparametrize $M$ by

$$
p(r ; x)=\exp \left[x^{1} f_{1}(r)+x^{2} f_{2}(r)-\psi(x)\right],
$$

where

and

$$
x^{1}=\frac{\mu}{\sigma^{2}}, \quad x^{2}=\frac{1}{2 \sigma^{2}}
$$

$$
\begin{gathered}
f_{1}(r)=r, \quad f_{2}(r)=-r^{2}, \\
\psi(x)=\frac{\mu^{2}}{2 \sigma^{2}}+\ln (\sqrt{2 \pi} \sigma)=\frac{\left(x^{1}\right)^{2}}{4 x^{2}}+\ln \sqrt{\frac{\pi}{x^{2}}} .
\end{gathered}
$$

Thus $M$ is an exponential manifold in $\mathcal{P}$. The induced Riemannian metric $L=g_{i j}(x) y^{i} y^{j}$ of an $f$-divergence on $M$ is given by

$$
g_{11}=\frac{\partial^{2} \psi}{\partial x^{1} \partial x^{1}}, \quad g_{12}=\frac{\partial^{2} \psi}{\partial x^{1} \partial x^{2}}, \quad g_{22}=\frac{\partial^{2} \psi}{\partial x^{2} \partial x^{2}} .
$$

The Gauss curvature of $L$ is a negative constant $K=-\frac{1}{2}$. 
Example 13.5 Let $M$ be the family of gamma distributions with event space $\Omega=\mathrm{R}^{+}$and parameters $\tau, \nu \in \mathrm{R}^{+}$which are defined by

$$
p(r ; \tau, \nu)=\left(\frac{\nu}{\tau}\right)^{\nu} \frac{r^{\nu-1}}{\Gamma(\nu)} \exp \left[-\frac{r \nu}{\tau}\right]
$$

where $\Gamma$ is the gamma function defined by

$$
\Gamma(\nu)=\int_{0}^{\infty} s^{\nu-1} e^{-s} d s .
$$

Note that $\tau=\langle r\rangle$ is the mean and $\tau^{2} / \nu=\operatorname{Var}(r)$ is the variance. Thus the coefficient of variation $\sqrt{\operatorname{Var}(r)} / \tau=1 / \sqrt{\nu}$ is independent of the mean.

Let $\mu:=\nu / \tau$. Then gamma distributions can be expressed by

$$
p(r ; \mu, \nu)=\exp [-\mu r+\nu \ln r-\ln r-\psi(\mu, \nu)],
$$

where

$$
\psi(\mu, \nu):=\ln \Gamma(\nu)-\nu \ln \mu .
$$

Thus $M$ is an exponential manifold in $\mathcal{P}$. See $[\mathrm{HH}]$ for related discussion.

Let $L$ be the induced Riemannian metric by any $f$-divergence. In the coordinate system $(\tau, \nu)$,

$$
g_{11}=\frac{\nu}{\tau^{2}}, \quad g_{12}=0=g_{21}, \quad g_{22}=\Psi^{\prime}(\nu)-\frac{1}{\nu},
$$

where $\Psi(\nu):=\Gamma^{\prime}(\nu) / \Gamma(\nu)$ is the logarithmic derivative of the gamma function. Since $\Psi(\nu)$ satisfies

$$
\frac{1}{2 \nu^{2}} \leq \Psi^{\prime}(\nu)-\frac{1}{\nu} \leq \frac{1}{\nu^{2}} .
$$

We have

$$
L_{1}:=\frac{\nu}{\tau^{2}} u^{2}+\frac{1}{2 \nu^{2}} v^{2}<L<\frac{\nu}{\tau^{2}} u^{2}+\frac{1}{2 \nu^{2}} v^{2}:=L_{2} .
$$

The Gauss curvature $K_{i}$ of $L_{i}$ and the Gauss curvature $K$ of $L$ are given

$$
K_{1}=-\frac{1}{2}<K=\frac{\Psi^{\prime}(\nu)+\Psi^{\prime \prime}(\nu) \nu}{4 \nu^{2}\left(\Psi^{\prime}(\nu)-1 / \nu\right)^{2}}<-\frac{1}{4}=K_{2} .
$$

The reader is referred to [DoMa] for the geometry of Gamma distributions and its applications.

\section{Duality of $f$-divergences}

Let $(\mathcal{P}, \mathcal{D})$ be a divergence space $(\mathcal{P}, \mathcal{D})$. By definition, the dual divergence $D^{*}$ is defined by

$$
\mathcal{D}^{*}(p, q):=\mathcal{D}(q, p), \quad p, q \in \mathcal{P} .
$$


Given a convex function $f:(0, \infty) \rightarrow \mathrm{R}$ with $f(1)=0$ and $f^{\prime \prime}(1)=1$. Let

$$
f^{*}(t):=t f\left(\frac{1}{t}\right), \quad t>0 .
$$

Then $f^{*}(t)$ satisfies that $f^{*}(1)=0$ and $f^{*^{\prime \prime}}(1)=f^{\prime \prime}(1)=1$. Let $\rho:=3+2 f^{\prime \prime \prime}(1)$ and $\rho^{*}:=3+2 f^{*^{\prime \prime \prime}}(1)$. We have

$$
\rho+\rho^{*}=0 .
$$

Note that

$$
\left(D_{f}\right)^{*}(p, q):=D_{f}(q, p)=D_{f^{*}}(p, q) .
$$

Thus $D_{f^{*}}$ is dual to $D_{f}$. By the above argument, $\left(D_{f}\right)^{*}=D_{f^{*}}$ induces an information structure

$$
\left(L_{f^{*}}, H_{f^{*}}\right)=\left(L, \rho^{*} N\right)=(L,-\rho N) .
$$

That is, $L_{f^{*}}(x, y)=L_{f}(x,-y)$ and $H_{f^{*}}(x, y)=H_{f}(x,-y)$. The information structure of $\left(D_{f}\right)^{*}$ is dual to that of $D_{f}$. In this sense, $D_{f}$ is said to be dualistic.

According to Lemmas 10.1 and 10.2, we have the following

Proposition 14.1 The information structure $\left(L_{f}, H_{f}\right)$ is $\alpha$-flat if and only if the dual structure $\left(L_{f^{*}}, H_{f^{*}}\right)=\left(L_{f}(x,-y), H_{f}(x,-y)\right)$ is $\alpha$-flat.

Let $f_{\rho}$ be the function defined in (4). Let $D_{\rho}:=D_{f_{\rho}}$. It is easy to see that

$$
\left(f_{\rho}\right)^{*}(t)=f_{-\rho}(t) .
$$

Thus

$$
\left(D_{\rho}\right)^{*}(p, q)=D_{\rho}(q, p)=D_{-\rho}(p, q)
$$

For $\rho \neq \pm 1$

$$
D_{\rho}(p, q)=\frac{4}{1-\rho^{2}}\left\{1-\int p(r)^{(1-\rho) / 2} q(r)^{(1+\rho) / 2} d r\right\},
$$

for $\rho= \pm 1$,

$$
D_{-1}(p, q)=D_{+1}(q, p)=\int p(r) \ln \frac{p(r)}{q(r)} d r .
$$

\section{References}

[AmNa] S.-I. Amari and H. Nagaoka, Methods of Information Geometry, Oxford University Press and Amer. Math. Soc. (2000).

[BaChSh] D. Bao, S.S. Chern and Z. Shen, An introduction to RiemannFinsler geometry, Springer, 2000.

[Am] S.-I. Amari, Differential Geometrical Methods in Statistics, Springer Lecture Notes in Statistics, 20, Springer, 2002. 
[DoMa] C.T.J. Dodson and H. Matsuzoe, An affine embedding of the gamma manifold, preprint.

[DzCo1] E.D. Dzhafarov and H. Colonius, Fechnerian metrics in unidimensional and multidimensional stimulus, Psychological Bulletin and Review, 6(1999), 239-268.

[DzCo2] E.D. Dzhafarov and H. Colonius, Fechnerian scaling, probabilitydistance hypothesis, and Thurstonian link. Technical Report \#45, Purdue Mathematical Psychology Program.

[DzCo3] E.D. Dzhafarov and H. Colonius, Fechnerian metrics, In P.R. Kileen \& W.R. Uttal (Eds.), Looking Back: The End of the 20th Century Psychophysics (pp. 111-116). Tempe, AZ: Arizona University Press.

[HH] T.-Y. Hwang and C.-Y. Hu, On a characterization of the gamma distribution: The independence of the sample mean and the sample coefficient of variation, Annals Inst. Statist. Math. 51(1999), 749753.

[La] S.L. Lauritzen, Statistical manifolds, Differential Geometry in Statistical Inferences, IMS Lecture Notes Monograph Series, 10, 96163, Hayward California, 1987.

[MuRi] M.K. Murray and J.W. Rice, Differential Geometry and Statistics, London: Chapman \& Hall, 1995.

[NaAm] H. Nagaoka and S. Amari, Differential geometry of smooth families of probability distributions, METR, 82-7.

[Sh1] Z. Shen, Differential Geometry of Spray and Finsler Spaces, Kluwer Academic Publishers, Dordrecht, 2001.

[SY] Z. Shen and G. C. Yildirim, A characterization of Funk metrics, preprint, 2005.

Math Dept, IUPUI, 402 N. Blackford Street, Indianapolis, IN 46202-3216, USA.

zshen@math.iupui.edu 